\title{
Listenkonstruktionen als vorgeformte Strukturen - Vorgeformte Ausdrücke innerhalb von Listenkonstruktionen
}

\author{
Heike Knerich (Bielefeld)
}

\begin{abstract}
In this paper lists are understood as complex pre-patterned structures. List constructions are composed of a number of elements which are embedded in a prefatory and a terminal utterance (cf. Jefferson 1990, Selting 2004). This complex pre-patterned structure may additionally contain smaller pre-patterned elements. On the basis of a corpus of authentic dyadic doctor-patient interviews I investigate the form and the functions in patients" use of list constructions while talking about fear and anxiety. The analysis is based on a broad concept of pre-patterned structures (cf. Gülich 2007), the method is a linguistic microanalysis that draws on ethnomethodological conversation analysis. In conclusion, I will discuss list structures with respect to different concepts of prefabricated linguistic and communicative units.
\end{abstract}

\section{$1 \quad$ Einleitung}

In den Gesprächen, die dieser Untersuchung zugrunde liegen, sind die PatientInnen mit der konversationellen Aufgabe konfrontiert, einem Arzt oder einer Ärztin ihre Anfallserkrankung und ihre damit zusammenhängenden Ängste zu schildern. Eine Formulierungsressource, auf die sie dabei zurückgreifen, sind vorgeformte Strukturen (cf. Gülich 2007, Knerich i. Vorb.). Dieser Begriff umfasst sowohl sozial geteilte, kulturell geprägte sprachliche Formen, also Phraseologismen und formelhafte Wendungen, als auch individuell verfestigte Redeweisen, auf die einzelne SprecherInnen immer wieder zurückgreifen. Vorgeformte Strukturen können unterschiedlich komplex sein, sie reichen von Wortverbindungen über satzförmige Strukturen bis zu komplexeren Einheiten wie Listenkonstruktionen und kommunikativen Gattungen (cf. Dausendschön-Gay et al. 2007, Gülich/Krafft 1998, Gülich 2007).

Mit der Einbeziehung komplexer Einheiten fasst das Konzept der Vorgeformtheit seinen Gegenstandsbereich sehr weit. Dies überschneidet sich mit neueren Entwicklungen in der Phraseologieforschung (cf. z. B. Schmale 2011), für die ebenfalls eine Erweiterung des Gegenstandsbereichs festgestellt werden kann. Dennoch steht hier eher die Beschreibung lexikalisierter Phraseme und vielfach deren Klassifikation im Zentrum (cf. Burger 2007). Einige Arbeiten befassen sich auch mit der Prozessierung lexikalisierter Phraseme im Gespräch (cf. Schmale 1999; Schmale 2012). Das Konzept der Orientierung an vorgeformten Strukturen, das der hier vorgenommenen qualitativ-empirischen Analyse authentischer institutioneller Gespräche zugrunde liegt, stellt Vorgeformtes als verfestigte Lösung von 
Formulierungsproblemen und kommunikativen Problemen ins Zentrum. Dabei geht es darum, Formen und Funktionen vorgeformter Strukturen im Kontext von Formulierungsarbeit in der Interaktion zu beschreiben (cf. Dausendschön-Gay et al. 2007b; Gülich 2007). Welche Gegenstände unter das Konzept der Vorgeformtheit fallen können, wird dabei durchaus diskutiert; so ist in der Gesprächsforschung nicht unumstritten, inwieweit neben verfestigten Ausdrücken und Wendungen auch auf der sprachlichen Oberfläche verfestigte komplexere Strukturen und sogar komplex musterhafte kommunikative Gattungen mit diesem Begriff gefasst werden können (cf. Gülich 2007; Knerich i. Vorb.; Günthner 2006, Quasthoff 2010). Auf diese Diskussion werde ich auf der Grundlage meiner folgenden Analysen von Listenkonstruktionen als vorgeformten Strukturen abschließend zurückkommen.

Alle hier analysierten Beispiele stammen aus einem Korpus von 20 authentischen dyadischen Arzt-Patient-Gesprächen, für dessen Zusammenstellung ich auf zwei interdisziplinär erhobene Korpora zurückgreifen durfte. ${ }^{1}$ Die PatientInnen leiden alle unter Angst im Zusammenhang mit Anfällen, seien es Panikattacken, epileptische oder dissoziative Anfälle. Die Gespräche liegen als Video- oder Audioaufnahmen vor und sind nach den GAT-Konventionen (Selting et al. 1998) transkribiert. Der methodische Zugang zu den Daten ist empirisch-explorativ und orientiert sich an der gesprächsanalytischen - besonders der konversationsanalytischen Methodologie (cf. z. B. Bergmann 1994, zur linguistischen Konversationsanalyse als Methode der Untersuchung von Formulierungsaktivitäten cf. Gülich 1994). In einer zyklischen Herangehensweise von Analyse und Datenexploration habe ich in etwas mehr als zwölf Stunden Material ca. 50 Aufzählungen ${ }^{2}$ identifiziert; die meisten davon werden mit rahmenden Elementen verwendet, so dass man von Musterhaftigkeit bzw. Vorgeformtheit sprechen kann $^{3}$ (cf. auch Jefferson 1990, Selting 2004). Feinanalytisch untersucht habe ich zunächst besonders die Listenkonstruktionen, die die PatientInnen im Kontext des Sprechens über Angst und Anfälle verwenden, später habe ich ergänzend thematisch anders gelagerte Listenkonstruktionen hinzugezogen.

Nach der Klärung der verwendeten Terminologie und methodischen Vorgehensweise stelle ich anhand der Feinanalyse eines Beispiels aus diesem Korpus dar, inwiefern ich Listenkonstruktionen als vorgeformte Strukturen verstehe, und zeige die Grundstruktur von Listenkonstruktionen auf. Anschließend beschreibe ich anhand von zwei Fallbeispielen, wie sich die Gesprächsbeteiligten gemeinsam an dieser Struktur orientieren. Zum Abschluss präsentiere ich den in meinem Korpus vorkommenden Sonderfall von Listenkonstruktionen mit inszenierender Funktion, bei dem auch kleinere konventionell vorgeformte Ausdrücke eine Rolle spielen. Im Fazit greife ich vor dem Hintergrund meiner Ergebnisse die oben

\footnotetext{
1 Die Gespräche entstammen zum einen dem Korpus der ZiF-Kooperationsgruppe "Kommunikative Darstellung und klinische Repräsentation von Angst - Exemplarische Untersuchungen zur Bedeutung von Affekten bei Patienten mit Anfallskrankheiten und/oder Angsterkrankungen" (Bergmann/Gülich/Schöndienst/Wörmann), zum anderen dem Korpus des DFG-Projekts "Linguistische Differenzialtypologie epileptischer und anderer anfallsartiger Störungen - diagnostische und therapeutische Aspekte" (Gülich/Schöndienst). Cf. für weitere Informationen www.unibielefeld.de/lili/forschung/projekte/epiling, www.uni-bielefeld.de/ZIF/KG/2004Angst/index.html.

2 Ich verwende den Begriff "Aufzählung" für die "eigentliche Liste", d.h. den Mittelteil der Listenkonstruktion. Selting (2000) dagegen differenziert zwischen einem allgemeineren Begriff "Aufzählung"/"listenartige Aufzählung", der prosodisch, syntaktisch und semantisch parallel formulierte "Listen" umfasst (cf. Selting 2000: 17), Selting (2004) wiederum verwendet die Begriffe Liste und Aufzählung synonym (cf. Selting 2004: 2, 37).

3 Im Korpus finden sich auch einige wenige Aufzählungen, die nicht einleitend und abschließend gerahmt sind. Diese habe ich in meine Untersuchung nicht einbezogen. Im hier untersuchten Korpus nutzen SprecherInnen solche Aufzählungen vor allem im Rahmen von Erzählungen.
} 
angesprochene Frage nach dem Gegenstandsbereich des Konzepts der Vorgeformtheit wieder auf.

\section{Listenkonstruktionen als vorgeformte Strukturen}

Unter einer Listenkonstruktion verstehe ich eine einleitend und abschließend gerahmte Aufzählung (cf. Selting 2004), die als Einheit von den umgebenden Äußerungen abgegrenzt ist und eine verfestigte Oberflächenstruktur aufweist. Diese wird in erster Linie mit prosodischen und zumeist auch mit syntaktischen Mittel hergestellt, weitere Ebenen der Verfestigung sind optional (cf. Knerich i. Vorb.). Damit sind Listenkonstruktionen "größere Strukturen", d.h. sie umfassen eine Folge von Turnkonstruktionseinheiten (TCU, cf. Sacks et al. 1974). Nach dem Konzept der vorgeformten Strukturen (cf. Dausendschön-Gay et al. 2007, Gülich/Krafft 1998, Gülich 2007), können Listenkonstruktionen insofern als vorgeformt verstanden werden, als sie verfestigte sprachliche Einheiten bilden.

Der Begriff der vorgeformten Strukturen, auf den ich mich hier beziehe, ist in der konversationsanalytischen Auseinandersetzung mit Interaktionsdaten entwickelt worden (cf. Gülich/Krafft 1992, 1997a, b, 1998; Dausendschön-Gay et al. 2007). Er beinhaltet die klassischen Phänomene der Phraseologie, erweitert diesen Gegenstandsbereich jedoch um größere Strukturen ${ }^{4}$ und um individuell geprägte Redeweisen ${ }^{5}$. Die methodische Herangehensweise der linguistischen Gesprächsforschung bzw. der Konversationsanalyse, auf deren Basis der Begriff entwickelt wurde, ermöglicht zu beobachten, wie InteraktantInnen Vorgeformtes im Formulierungs- und Interaktionsprozess verwenden (cf. Gülich 2007: 59, Knerich i. Vorb.). Durch die Rekonstruktion solcher Formulierungs- und Interaktionsprozesse lässt sich auch zeigen, wie Interagierende sich an verfestigten Mustern orientieren, die größer sind als formelhafte Ausdrücke oder Wendungen.

Das folgende Beispiel führt Listenkonstruktionen als eine von den umgebenden Äußerungen abgegrenzte Einheit ein, die aus einer Aufzählung von Elementen und einer einleitenden sowie abschließenden Äußerung besteht. Ich betrachte den Ausschnitt zunächst sequenzanalytisch und stelle die Listenkonstruktion anschließend schematisch dar. In Bezug auf die Ergebnisse von Jefferson (1990) und Selting (2004) zeige ich dann an diesem Beispiel die Grundstruktur von Listenkonstruktionen auf und beziehe diese strukturelle Vorgeformtheit auf Konzepte aus der Phraseologieforschung.

\footnotetext{
4 Forschung, die sich mit verfestigten sprachlichen bzw. kommunikativen Elementen befasst, geht insgesamt dazu über, größere Strukturen einzubeziehen: Imo (2007) weist darauf hin, dass es in der Konstruktionsgrammatik Überlegungen gibt, Konstruktionen auf Text- bzw. Diskursebene einzubeziehen (cf. Imo 2007: 24), Günthner (2006) fasst grammatische Konstruktionen und kommunikative Gattungen unter dem Oberbegriff 'Vorgeformtheit' zusammen, Schmale (2011) schlägt den Begriff "präformierte Konstruktionseinheiten" vor, unter den auch kommunikative Gattungen gefasst werden (cf. Schmale 2011: 180-181, 188).

5 Zur Forschungsgeschichte und zum Zusammenhang mit der Phraseologieforschung cf. Gülich/Krafft 1998: 11-15.
} 


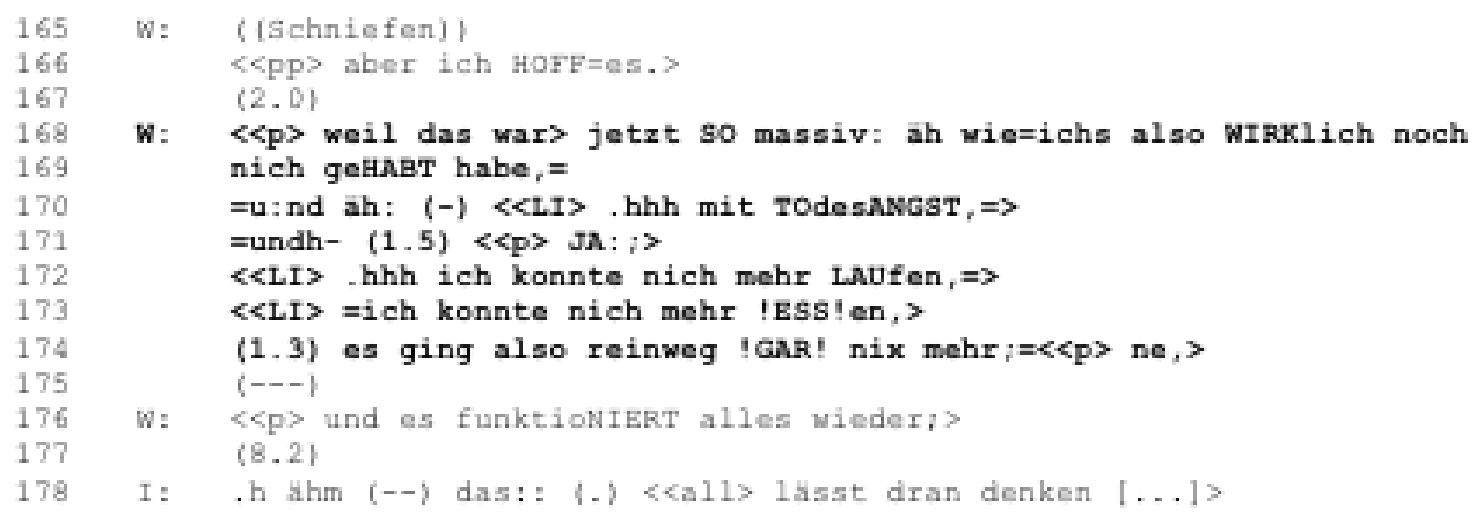

Ausschnitt 1: ich konnte nich mehr LAUfen,..., - Frau Wiesinger, Z. 165-17

\section{Sprechersiglen: $\mathrm{W}=$ Frau Wiesinger; $\mathrm{A}=$ Arzt $1 . \mathrm{LI}=$ Listenintonation6}

Als hochstufende Einleitung verwendet Frau Wiesinger eine individuell rekurrente Formulierung: (das war) so massiv; dabei fungiert die kausale Konjunktion an dieser Stelle als Diskursmarker, der die Fortsetzung und den Ausbau der bisherigen Ausführungen signalisiert. Eine Fortsetzung wird vor allem durch die Prosodie projiziert, zudem durch das als kataphorische Proform; auch die Bewertung und Hochstufung SO massiv und der Vergleich wie=ichs also WIRKlich noch nich geHABT habe (Z. 168-169) haben eine gewisse projizierende Kraft.

Den nächsten Turn beginnt Frau Wiesinger mit der Konjunktion u:nd, nach einem Verzögerungssignal und einer Pause nennt sie mit TOdesANGST, (Z. 170). Diese Turnkonstruktionseinheit ist durch die Intonation als Listenelement ausgewiesen. Mit dem additiven Anschluss zeigt Frau Wiesinger, dass sie ihre Äußerung fortsetzen wird. Es folgt eine lange Pause und ein leise und gedehnt gesprochenes $J A$;, beides weist auf Formulierungsschwierigkeiten hin (Z. 171).

In den folgenden beiden Turns zählt Frau Wiesinger dann Handlungseinschränkungen infolge der Panikerkrankung auf; durch die Prosodie sind beide Turns als Listenelemente kontextualisiert (Z. 172-173). Mit der abschließend rahmenden konventionell vorgeformten Wendung es ging also reinweg !GAR! nix mehr; (Z.174) wird nach einer Pause die Listenkonstruktion abgeschlossen. Die Wendung "es ging gar nichts mehr" findet sich nicht in phraseologischen Wörterbüchern ${ }^{7}$, sie wird aber von Analysegruppen ${ }^{8}$ als vorgeformt

\footnotetext{
6 Ich verwende den Begriff Listenintonation als Oberbegriff für eine Intonation, die bestimmte Turns als Listenelemente ausweist (cf. z. B. auch Günthner 2012: 9). Selting (2000, 2004) beschreibt verschiedene Formen der Listenintonation.

${ }^{7}$ In einer kursorischen Suchmaschinenabfrage findet sich die Wendung vorwiegend im Präsens. Etliche in der mündlichen Kommunikation verfestigte Wendungen finden sich (noch) nicht in Lexika, obwohl sie von Mitgliedern der Sprachgemeinschaft als solche erkannt werden und/oder in kursorischen Suchmaschinenabfragen gehäuft zu finden sind. Kallmeyer/Keim (1986) stellen für eine kleinere Sprechergruppe fest: "[...] der fortgesetzte Prozess der Formelprägung ist fester Bestandteil unserer alltäglichen Sprachpraxis" (cf. Kallmeyer/Keim 1986: 102). Der Prozess der Phraseologisierung lässt sich auch an den Beispielen von Schmale (1999) nachvollziehen: Zwei Äußerungen in Fernseh-Talkshows werden aufgrund ihrer semantischen Unregelmäßigkeit in Bezug auf den Kontext als Kandidaten für "Neuschöpfungen vorgeformter Sequenzen" eingestuft, die zum Zeitpunkt der Untersuchung nicht in den Wörterbüchern standen, die zum Abgleich ihrer Lexikalisierung herangezogen wurden (cf. Schmale 1999: 167). Beide von Schmale (1999) untersuchten Wendungen finden sich heute als sozial geteilte vorgeformte Ausdrücke in neueren Lexika. Zu methodischen Implikationen cf. Knerich i. Vorb.

${ }^{8} \mathrm{Zu}$ Analysegruppen als Mittel der Triangulation cf. Knerich (i. Vorb.).
} 
bestätigt. Weitere Beendigungsmarkierungen sind hier zudem die fallende Intonation und das leiser gesprochene ne, in Tag-Position (Z. 174). Der Arzt übernimmt hier dennoch nicht das Rederecht, woraufhin Frau Wiesinger mit und es funktioNIERT alles wieder; erneut auf ihren nach der Behandlung deutlich verbesserten Zustand hinweist und mit diesem Rückverweis zugleich die gesamte Sequenz abschließend rahmt (Z. 176). Nach einer sehr langen Gap übernimmt dann der Arzt das Rederecht.

Es handelt sich hier um eine Aufzählung von drei Elementen, die von zwei vorgeformten Wendungen gerahmt ist, einer individuell rekurrenten und einer sozial geteilten. Dabei ist ein Wechsel in der Gestaltung der Listenelemente zu beobachten: Das gedehnt gesprochene $J A$ :; in Z. 171 fungiert als Gelenkstelle, nach der die mit TOdesANGST begonnene Liste in eine individuell rekurrente Liste umkonstruiert wird (cf. Knerich i. Vorb.). Dabei ist der erste nicht vorgeformte - Teil der Liste reich an Verzögerungsphänomenen, während die beiden vorgeformten Listenelemente schnell gesprochen sind und keine Verzögerungsmarkierungen enthalten. Diese beiden Elemente benennen Auswirkungen der Angsterkrankung: ich konnte nich mehr LAUfen, ich konnte nich mehr !ESS!en, (Z. 172-173); inhaltlich handelt es sich um gravierende Einschränkungen zum einen der Fortbewegung, zum anderen der überlebenswichtigen Fähigkeit zu essen. Mit diesen Listenelementen weist Frau Wiesinger ihren Zustand in der Panikerkrankung bereits inhaltlich als einen höchst dramatischen aus. Mit es ging also reinweg!GAR! nix mehr; (Z. 174) fasst sie dies zusammen und bringt es auf den Punkt, generalisiert es und stuft es erneut hoch.

Diese vorgeformte Wendung schließt die Listenkonstruktion ab; zugleich hat sie das Potenzial, das Thema zu beenden wie auch interaktiv-gesprächsstrukturierend die Sequenz abzuschließen und das Rederecht an den Gesprächspartner zu übergeben. Der Arzt übernimmt es hier jedoch nicht direkt, auch Frau Wiesinger spricht nicht weiter; so entsteht eine lange Gap.

Schematisch kann diese Listenkonstruktion wie folgt als zweifache Dreierstruktur (cf. Selting 2004: 3-4, siehe auch unten) dargestellt werden:

\begin{tabular}{|c|c|c|c|}
\hline \multicolumn{2}{|c|}{ I. einleitender Rahmen } & \multirow{2}{*}{$\begin{array}{l}\text { weil das war jetzt SO massiv: äh wie=ichs } \\
\text { also WIRKlich noch nich geHABT habe, } \\
=\text { uc:nd äh: }(-)<<L I>\text {, hhh mit } \\
\text { TOdesANGST, }=>\end{array}$} & \multirow{2}{*}{$\begin{array}{l}\text { individuell vorgeformter } \\
\text { Ausdruck so massiv }\end{array}$} \\
\hline II. Aufzählung & 1. Listenelement & & \\
\hline & 2. Listenelement & $\begin{array}{l}<<L l>\text {, hhh ich konnte nich mehr } \\
L A U \text { fen, }=>\end{array}$ & \\
\hline & 3. Listenelement & $<<L l>=i c h$ konnte nich mehr !ESS!en, $>$ & \\
\hline \multicolumn{2}{|c|}{ III. abschließender Rahmen } & es ging also reinweg ! GAR! nix mehr:= ne, & $\begin{array}{l}\text { individuell } \\
\text { rekurrente/konventionell } \\
\text { vorgeformte Wendung es } \\
\text { ging gar nichts mehr }\end{array}$ \\
\hline
\end{tabular}

Schematische Darstellung 1a: Listenkonstruktion ich konnte nich mehr LAUfen, ... (Frau Wiesinger)

Frau Wiesinger füllt diese zweifache Dreierstruktur mit auf die Panik bezogenen Inhalten; dabei sind die Listenelemente inhaltlich an der Einschränkung grundlegender Handlungen orientiert. Die sozial geteilte Wendung steht an der an funktionell relevanten abschließenden Position. Insgesamt ist diese Listenkonstruktion die Vollform eines individuell vorgeformten Modells (cf. Knerich i. Vorb.): Die Patientin greift in diesem Gespräch wiederholt auf formal 
und inhaltlich ähnlich gestaltete Listenkonstruktionen zurück, wenn sie darstellt, wie ihre Handlungsmöglichkeiten durch die Panikerkrankung eingeschränkt wurden.

Unter Listenkonstruktionen verstehe ich also eine - vor allem durch die Prosodie zusammengehaltene - Konstruktion aus einer Aufzählung von $n$ Elementen, die einleitend und abschließend gerahmt ist. Die rahmenden Elemente haben einen inhaltlichen Bezug zueinander. Dabei können an jeder Stelle der Konstruktion vorgeformte Ausdrücke und Wendungen stehen, funktionell besonders interessant sind die Positionen des Rahmens und des letzten Listenelements.

Eine solche Listenkonstruktion umfasst ganz offensichtlich mehr als eine Turnkonstruktionseinheit (cf. Sacks et al. 1974), und sie ist auch keine Konstruktion im engeren konstruktionsgrammatischen Sinne (zusammenfassend $\mathrm{zu}$ diesem Konzept cf. Günthner 2006: o. S., Imo 2007: 24-25; Schmale 2011: 184-185). Es handelt sich aber um eine Einheit, die eine erkennbare Oberflächenstruktur hat und an der sich - auch wenn sie zumeist von einer Person produziert wird - nachweislich alle am Gespräch Beteiligten orientieren (cf. Selting 2004: 8-15; Abschnitt 2). Dies sind Hinweise darauf, dass die gesamte Einheit als vorgeformtes Modell konzipiert werden kann (zur Orientierung am Modell cf. Dausendschön-Gay et al. 2007: 183-189). Dieser Frage werde ich anhand der bisherigen Beschreibungen von Listenkonstruktionen und anhand weiterer Fallanalysen nun genauer nachgehen.

Selting (2004) zeigt anhand von Interaktionsdaten, dass eine einleitend und abschließend gerahmte Aufzählung eine Gesamtstruktur bildet, die sie als "Drei-Komponenten-Struktur" bezeichnet. Sie ist wie folgt aufgebaut (1): Eine "Projektionskomponente" rahmt die Liste einleitend, (2) es folgen $n$ Listenelemente, (3) eine "Gestaltschließungskomponente", die meist eine anaphorische Verbindung zur Projektionskomponente hat, rahmt die Liste abschließend (cf. Selting 2004: 3-8). Für die einleitende Projektionskomponente differenziert Selting (2004) zwei mögliche Formen: eine "Prä-Detaillierungskomponente", mit der die InteraktantInnen mit kataphorischen Ausdrücken die folgende Darstellung projizieren, und eine allgemeinere Formulierung, mit der eine Elaboration, Explikation, Exemplifikation, Illustration oder Ähnliches angekündigt wird (Selting 2004: 7). Oftmals sind derartige Listenkonstruktionen zudem noch in größere Einheiten eingebettet; dann haben sie nach Selting die Funktion, innerhalb solcher größeren narrativen, explanativen, argumentativen etc. Strukturen bestimmte Aspekte zu detaillieren (Selting 2004: 7).

Für den Mittelteil, die eigentliche Aufzählung, weist Jefferson (1990) für das Englische eine Präferenz für drei Elemente nach, die Selting (2004) für das Deutsche bestätigt. Für das dritte Listenelement verwenden Interagierende teilweise ein generalisierendes Abschlusselement, den generalized list completer (cf. Jefferson 1990: 67), entweder um eine Liste abzuschließen, bei der die Suche nach einem dritten Element scheitert, oder um als drittes Element einer Dreierliste anzuzeigen, dass es noch viele mögliche Elemente gibt (cf. Jefferson 1990: 67-68), und somit eine offene Liste zu konstruieren. Die Listenelemente selbst werden meist durch prosodische, semantische und syntaktische Parallelität als solche erkennbar gemacht. Listenelemente können aber auch prosodisch als solche markiert sein, wenn sie syntaktisch nicht völlig gleichförmig sind. Es ist also in erster Linie an der Prosodie erkennbar, dass 
Gesprächsbeteiligte die Ressource der Listenkonstruktion wählen (Selting 2004: 3-4; cf. auch Tannen 1987: 596-598).

Listenkonstruktionen können demnach als auf der Ebene der Oberflächenstruktur vorgeformt verstanden werden, wobei diese Struktur weitgehend von der Prosodie bestimmt wird. Hinzu kommen oftmals eine syntaktische Parallelität der einzelnen Listenelemente und eine spezifischere Ausgestaltung bestimmter funktional relevanter Positionen, z. B. ein verallgemeinerndes bzw. allgemeines letztes Listenelement (cf. Jefferson 1990, Knerich i. Vorb., Selting 2004).

Unter Rückgriff auf die Terminologie von Selting (2004), die Ergebnisse von Jefferson (1990) und Selting (2004) sowie die Überlegungen zur verfestigten Oberflächenstruktur ergibt sich folgende Grundstruktur für eine Listenkonstruktion:

\begin{tabular}{|c|c|c|c|}
\hline \multicolumn{2}{|c|}{ I. Projektionskomponente } & $\begin{array}{l}\text { weil das war jetzt SO massiv: äh } \\
\text { wie=ichs also WIRKlich nech nich } \\
\text { geHABT habe, = }\end{array}$ & $\begin{array}{l}\text { Ein einleitender Turn projiziert } \\
\text { die „eigentliche" Liste, d.h. die } \\
\text { Aufzăhlung von Elementen. }\end{array}$ \\
\hline \multirow[t]{3}{*}{ II. Liste } & 1. Listenelement & $\begin{array}{l}=\text { u.nd äh: }(\gamma<<<L \text {, hhh mit } \\
\text { TOdesANGST, }=>\end{array}$ & \multirow{3}{*}{$\begin{array}{l}\text { Es folgt eine Nennung von drei } \\
\text { inhaltlich zusammenhăngenden } \\
\text { Elementen, die zumindest } \\
\text { prosodisch als Liste markiert } \\
\text { sind. }\end{array}$} \\
\hline & 2. Listenelement & $\begin{array}{l}<\langle L\rangle>\text {, hhh ich konnte nich mehr } \\
L A U \text { fen, }=>\end{array}$ & \\
\hline & 3. Listenelement & $\begin{array}{l}<\langle L|>=\text { ich kannte nich mehr } \\
\text { IESS!en, }>\end{array}$ & \\
\hline \multicolumn{2}{|c|}{ III. GestaltschlieBungskomponente } & $\begin{array}{l}\text { es ging also reinweg ! GAR! nix mehr; = } \\
\text { ne, }\end{array}$ & $\begin{array}{l}\text { Die Konstruktion wird mit einer } \\
\text { abschlieBenden Äußerung } \\
\text { beendet, die zumeist einen } \\
\text { Bezug zur einleitenden } \\
\text { ÄuBerung hat (Rahmen). }\end{array}$ \\
\hline
\end{tabular}

Schematische Darstellung 1b: Grundstruktur einer Listenkonstruktion am Beispiel von Ausschnitt 1

Diese Art der hauptsächlich strukturellen Vorgeformtheit kann analog zu den von Burger (1982) beschriebenen "Modellbildungen" und den von Fleischer (1997) definierten Konzept der "Phraseoschablonen" gesehen werden. Modellbildungen sind ein

Typus von Wortverbindungen, bei dem ein bestimmtes syntaktisches Schema mit einer typisierten Semantik ausgestattet ist, wobei die lexikalisierte Besetzung der syntaktischen Positionen mehr oder weniger frei ist. Neben dem einfachen Fall "X hin, X her", der vermutlich beliebig lexikalisch ausfüllbar ist, gibt es Modelle wie "von X zu Y", hinter denen sich mehrere semantische Schemata verbergen können (Burger 1982: 35).

Solche syntaktischen Muster mit mehr oder weniger freien semantischen Festlegungen zählt Burger (1982, 1998) zum Bereich der Phraseologie; er geht zudem davon aus, dass die syntaktische Struktur eine bestimmte semantische Interpretation nahelegt (cf. Burger 1998: 42-43). Diese Bedeutung des Begriffs "Modellbildungen" wird im neueren Handbuch der Phraseologie allerdings nicht mehr aufgeführt (cf. Burger et al. 2007). Als "Phraseoschablonen" bezeichnet Fleischer (1997) syntaktische Konstruktionen mit einer "festgeprägten" Modellbedeutung, welche die Bedeutung der konkreten Form vorbestimmt. Er beschreibt sie als "nichtprädikative Wortverbindungen, deren lexikalische Füllung variabel ist, die aber eine Art syntaktischer Idiomatizität aufweisen" (Fleischer 1997: 135-136).

Die über prosodische und syntaktische Mittel hergestellte feste Struktur von Listenkonstruktionen ist solchen Konzepten aus der Phraseologie analog. Der Unterschied ist 
jedoch, dass Listenkonstruktionen größere Strukturen sind, die mit dem gesprächsanalytisch entwickelten Konzept von Vorgeformtheit als vergleichbar beschrieben werden können. Im Folgenden betrachte ich anhand von Beispielen aus meinem Korpus Abwandlungen von der hier dargestellten Grundstruktur einer gerahmten Liste von drei Elementen. Die Orientierung an der Struktur der Listenkonstruktion als Ganzes bleibt dabei erkennbar.

\section{Orientierung an der Gesamtstruktur}

Die beiden nun folgenden Beispiele weichen von der bisher beschriebenen Gesamtstruktur insofern ab, als im Mittelteil mehr als drei Elemente aufgezählt werden. Die interaktive Orientierung an der Gesamtstruktur ist im einem Fall daran zu erkennen, dass die Gesprächspartnerin in den Formulierungsprozess ihres Gegenübers mit einem Beendigungsversuch eingreift, im anderen Fall daran, dass eine Äußerung des Gesprächspartners als Ausgangspunkt für die Produktion der Listenkonstruktion genutzt wird. Diese interaktive Orientierung an der Gesamtstruktur kann besonders gut mit gesprächsanalytischen Mitteln beschrieben werden und ist ein Hinweis auf die Musterhaftigkeit der Listenkonstruktion.

\subsection{Orientierung an der Gesamtstruktur bei der Aufzählung von mehr als drei Elementen}

Es gibt verschiedene Möglichkeiten, mittels einer Listenkonstruktion auf eine große oder unendliche Anzahl von Elementen zu verweisen. Wenn SprecherInnen der Präferenz für drei Elemente folgen, können sie dafür ein generalisierendes letztes Listenelement verwenden, zum Beispiel "und so weiter" (Jefferson 1990: 66-67). Eine offene Liste kann aber auch ausschließlich über die Prosodie kontextualisiert werden (Selting 2004: 3-4; zur prosodischen Gestaltung von offenen im Vergleich zu geschlossenen Listen cf. Selting 2004: 15-34).

Im vorliegenden Korpus produzieren die Patientinnen darüber hinaus auch Listen mit mehr als drei Elementen, um im Zusammenhang mit panikartiger Angst Überwältigung durch eine große Anzahl oder Vielfalt von Gedanken oder Symptomen darzustellen. Die folgende Beispielsequenz stammt aus dem ersten Teil des Gesprächs mit Frau Spree, in dem sie der Ärztin selbstinitiiert die "schrecklichen Nächte mit Panikattacken" schildert, die ihrem Klinikaufenthalt vorausgingen. Zu diesem Zeitpunkt des Gesprächs hat Frau Spree in Bezug auf ihre Paniksymptome bereits zwischen tagsüber und nachts unterschieden und herausgestellt, dass die nächtliche Panik deutlich schlimmer war.

Der letzte Zugzwang, den die Ärztin vor diesem Ausschnitt gesetzt hatte, war die Frage danach, wie die schlimmeren Attacken des Nachts vorbeigegangen sind; Frau Spree hat daraufhin noch einmal betont, dass diese gar nicht vorübergingen und dass sie nicht im Bett bleiben konnte. Unmittelbar vor der zitierten Sequenz entwirft Frau Spree ein Szenario: Sie hätte sich hinlegen und liegen bleiben können, es hätte ja nicht viel passieren können, dies war ihr jedoch nicht möglich. Auf diese Unmöglichkeit, in den nächtlichen Attacken überhaupt etwas zu tun, bezieht sie sich zu Beginn des Ausschnitts (Z. 407-408): 


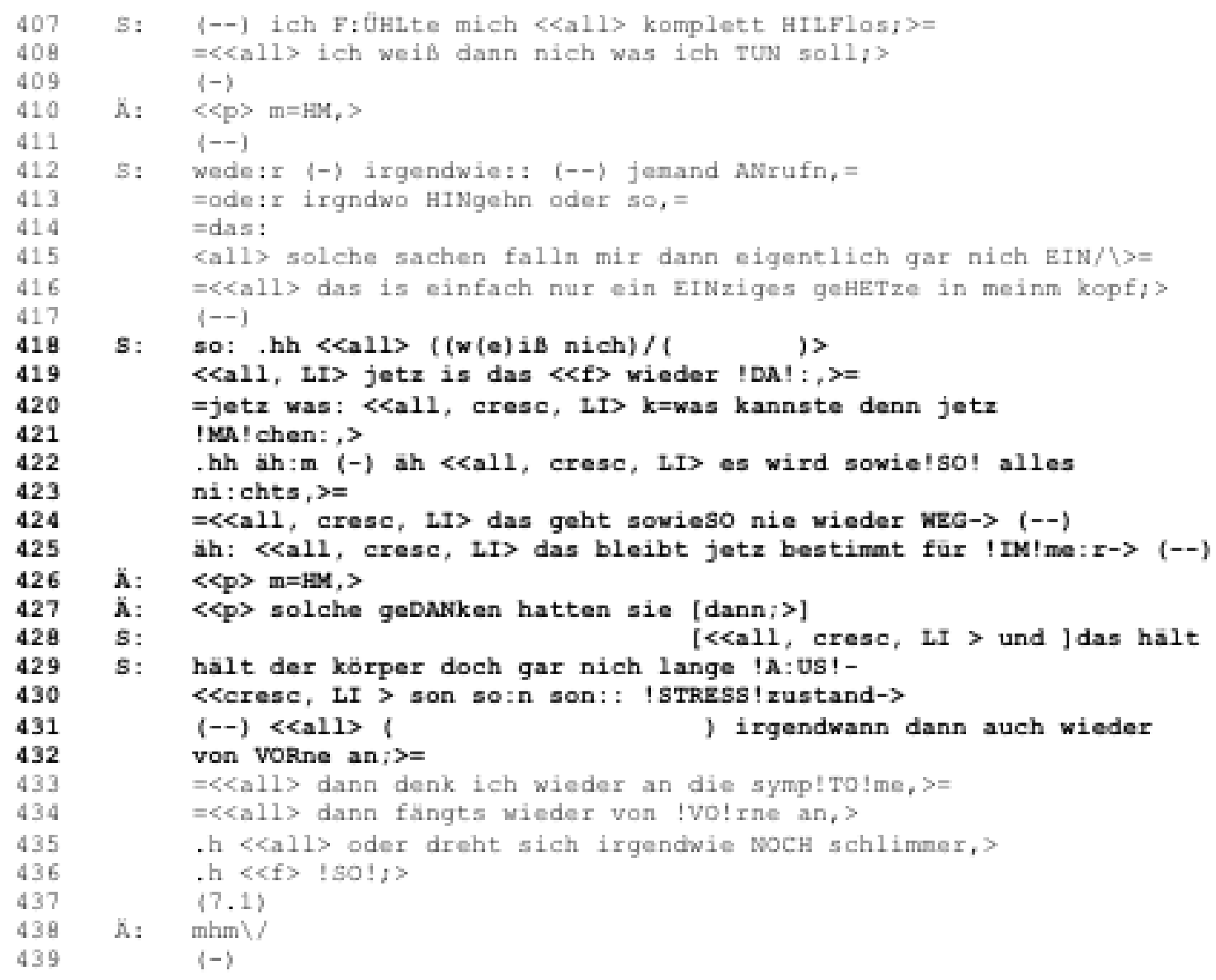

Ausschnitt 2: ein EINziges geHETze in meinm kopf - Frau Spree, Z. 407-439

Sprechersiglen: $\mathbf{S}=$ Frau Spree; $\ddot{\mathrm{A}}=\ddot{\text { Arztin. }} \mathbf{L I}=$ Listenintonation

Im Anschluss an die Betonung ihrer Hilflosigkeit wechselt Frau Spree ins Präsens (Z. 407) und beginnt damit eine verallgemeinernde Darstellung. Zunächst zählt sie Dinge auf, die sie tun könnte, die ihr aber in solchen Situation nicht in den Sinn kommen (Z. 412-415). Dann beschreibt sie ihren mentalen Zustand während dieser Panikattacken metaphorisch; dabei fällt die apersonale Agency auf, die zu der vorher beschriebenen Hilflosigkeit passt: Ihr als tätigem Subjekt fällt nichts ein (Z.415), trotzdem ist in ihrem Kopf einfach nur ein EINziges geHETze. Frau Spree formuliert dies hier also so, dass sie weder sich selbst noch der Krankheit Agentivität zuschreibt: Auch die Gedanken erscheinen hier nicht aktiv, sie passieren einfach (Z. 416).

Nach einer kürzeren Gap setzt Frau Spree mit einem gedehnten so fort, drückt dann aber im selben Turn zunächst Unsicherheit aus (Z. 418). Dieser Turn kann als Projektionskomponente für Redewiedergabe oder die Nennung von Beispielen beschrieben werden. Im Folgenden zählt sie dann Gedanken auf, die das "einzige Gehetze" ausmachen. Dass sie sich dabei an der Struktur der Liste orientiert, kontextualisiert sie über die Listenintonation; zudem bildet sie das Gehetze der Gedanken stimmlich durch die Schnelligkeit und die große oder ansteigende Lautstärke ab (Z. 419-425). 
Der erste zitierte Gedanke bezieht sich auf die Panik, auf die Frau Spree hier nur mit dem bestimmten Artikel verweist, und eine nicht näher bezeichnete aktuelle Situation, wie das Temporaladverb andeutet (jetz is das wieder !DA!:, Z. 419). Letzteres gilt auch für den zweiten Gedanken, eine Frage nach Handlungsmöglichkeiten (Z. 420-421). Der nächsten Gedanke, den Frau Spree nach einigen Verzögerungen formuliert, beantwortet die Frage nach Handlungsmöglichkeiten in einer verallgemeinernden und absoluten Form (alles, nichts) negativ (Z. 422-423). Dem folgen zwei weitere absolut formulierte Aussagen, die noch stärker verallgemeinern und die Aussichtslosigkeit der Situation betonen; dabei ist das fünfte Element eine Reformulierung des vierten (Z. 424-425). Auf diese beiden Listenelemente folgt jeweils eine Pause und sie enden in schwebender Intonation. Dies erzeugt eine zusätzliche Parallelität dieser beiden Elemente, die durch die schnelle und ansteigende Listenintonation dennoch klar als Teil der gerade produzierten Liste ausgewiesen sind.

In der zweiten Pause, d.h. nach dem fünften Listenelement, reagiert die Ärztin mit einem Rückmeldesignal (Z. 426); als Frau Spree an dieser Stelle nicht sofort fortsetzt, bietet sie dann mit leiser Stimme eine Gestaltschließung an: solche geDANken hatten sie dann; (Z. 427). Hier zeigt sich eine Orientierung der Gesprächspartnerin an der Drei-Komponenten-Struktur, wie Selting (2004) sie nachgewiesen hat. Frau Spree nimmt das Angebot der Ärztin jedoch weder strukturell noch inhaltlich auf, sondern setzt die Liste in der Überlappung fort. Dabei nimmt sie die starke Verallgemeinerung zurück und bezieht die Aussichtslosigkeit der Situation etwas konkreter auf die körperlichen Symptome (Z. 428-429). Dieser Turn ist prosodisch eindeutig als sechstes Listenelement gestaltet und an die beiden vorhergehenden Elemente angeschlossen. Auch der nächste Turn ist noch mit ansteigender Lautstärke und Listenintonation konstruiert. Die einzige prosodische Abweichung von den anderen Elementen besteht darin, dass dieses letzte Listenelement nicht mehr schnell gesprochen ist. Auch die Verzögerung durch die Wiederholung von son unterscheidet diesen Turn von den vorhergehenden (Z. 430).

Mit dem nächsten - zum Teil unverständlichen -, wieder schnell gesprochenen Turn schließt Frau Spree dann diese Liste von Gedanken in der Panik ab (() irgendwann dann auch wieder von VORne an;) und bezieht sie darauf zurück, dass sie die Panik als ununterbrochen wahrgenommen hat (Z.431-432). Die fallende Intonation weist hier eindeutig auf eine Gestaltschließung hin; Frau Spree fährt aber mit schnellem Anschluss fort mit einer präzisierenden Reformulierung (Z.433). Diese beiden Turns sind temporal eingeleitet und verweisen auf eine Chronologie, eine Entwicklung von Gedanken an die symp!TO!me, zu Gedanken an eine Panikattacke oder eine weitere Verschlimmerung. Frau Spree stellt hier also einen Zusammenhang zwischen Gedanken und Panikattacken her.

Frau Spree orientiert sich hier zum einen an einer Listenkonstruktion mit mehr als drei Elementen, zum anderen an der Drei-Komponenten-Struktur, der Rahmung der Liste durch eine Projektions- und eine Gestaltschließungskomponente, um innerhalb einer allgemeinen Darstellung der andauernden nächtlichen Panik das geHETze in ihrem Kopf zu detaillieren, zu veranschaulichen und zu (re-)inszenieren. Dass sich beide Gesprächspartnerinnen an dieser Struktur orientieren, wird an dem Versuch der Ärztin deutlich, in einer Pause nach dem fünften Listenelement die Gestaltschließung zu übernehmen. Frau Spree setzt ihre Liste jedoch fort und illustriert auch damit die Unzahl der Gedanken, die in der Panik auf sie einstürmen. In diesem interaktiven Zusammenspiel bildet sich auch die Hilflosigkeit 
angesichts dieser Gedanken ab, die - sowohl in den rekonstruierten Situationen als auch an diesem Punkt der Interaktion - weder von Frau Spree selbst noch von anderen anwesenden Personen unterbrochen werden können.

Schematisch kann diese Listenkonstruktion wie folgt dargestellt werden:

\begin{tabular}{|c|c|c|}
\hline \multicolumn{2}{|c|}{ I. Projektionskomponente } & so: $h h<<a l l>((w / e) i \beta$ nich $) /(\quad)$ \\
\hline \multirow[t]{5}{*}{ II. Liste } & 1. Listenelement & $<<$ all, $L>$ jelz is das $<<\beta$ wieder $! D A !:>>=$ \\
\hline & 2. Listenelement & $=$ jetz was: $\langle<a l l$, cresc, $L l\rangle k=$ was kannste denn jelz ! MAlchen: $\rangle$ \\
\hline & 3. Listenelement & hh äh:m $(-)$ äh $<<a l l$, cresc, $L I>$ es wird sewie!SO! alles ni.chts, $>=$ \\
\hline & 4. Listenelement & $=<<a l l$, cresc, $L I>$ das geht sowiesO nie wieder WEG $->(-)$ \\
\hline & 5. Listenelement & $a h t:<<$ all, cresc, $L I>$ das bleibt jetz bestimmt fitr !IM!me: $r>(-)$ \\
\hline \multicolumn{2}{|c|}{$\begin{array}{l}\text { III. Gestaltschließungskomponente } \\
\text { Angebot durch die Ärztin }\end{array}$} & $\ddot{A}:<<p\rangle m=H M\rangle,\langle<p\rangle$ solche geDANken hatlen sie [dann, $>]$ \\
\hline & 6. Listenelement & $\begin{array}{l}{[<<a l l, \text { cresc, } L I>\text { und }] \text { das hält hält der körper dach gar nich lange }} \\
\text { !A:US!- }\end{array}$ \\
\hline & 7. Listenelement & $\langle<$ cresc, $L I\rangle$ son so:n son:: ISTRESS!zustand -$\rangle$ \\
\hline \multicolumn{2}{|c|}{ III. Gestaltschließungskomponente } & $\begin{array}{l}(-)<<a l l>(\quad) \text { irgendwann dann auch wieder won VORne } \\
\text { an } ;>=\end{array}$ \\
\hline
\end{tabular}

Schematische Darstellung 2: Listenkonstruktion ein EINziges geHETze in meinm kopf (Frau Spree)

Dieses Beispiel einer Liste mit deutlich mehr als drei Elementen zeigt, wie InteraktantInnen sich an der komplexen vorgeformten Struktur der Listenkonstruktion orientieren und diese dabei ihren aktuellen Kommunikationszielen anpassen, indem sie im Mittelteil mehr als drei Elemente aufzählen. Dabei wird die Fortsetzung der Liste über die Prosodie kontextualisiert. Im Angebot der Gesprächspartnerin, die Konstruktion abzuschließen, wird erkennbar, dass diese sich an der von der Sprecherin projizierten Gesamtkonstruktion orientiert. Dass die Sprecherin dieses Angebot ihres Gegenübers nicht annimmt, ist für eine Interpretation in Bezug auf das Krankheitsgeschehen bedeutsam.

\subsection{Ko-Konstruktion der Gesamtstruktur}

Im folgenden Beispiel behandelt Frau Epang eine Frage des Arztes als Beginn einer Listenkonstruktion. Der Sequenz geht eine vom Arzt initiierte szenische Rekonstruktion des schlimmsten Angstanfalls durch die Patientin voraus. Nach einer Gap schließt der Arzt die folgende Frage nach Angstsymptomen an: 


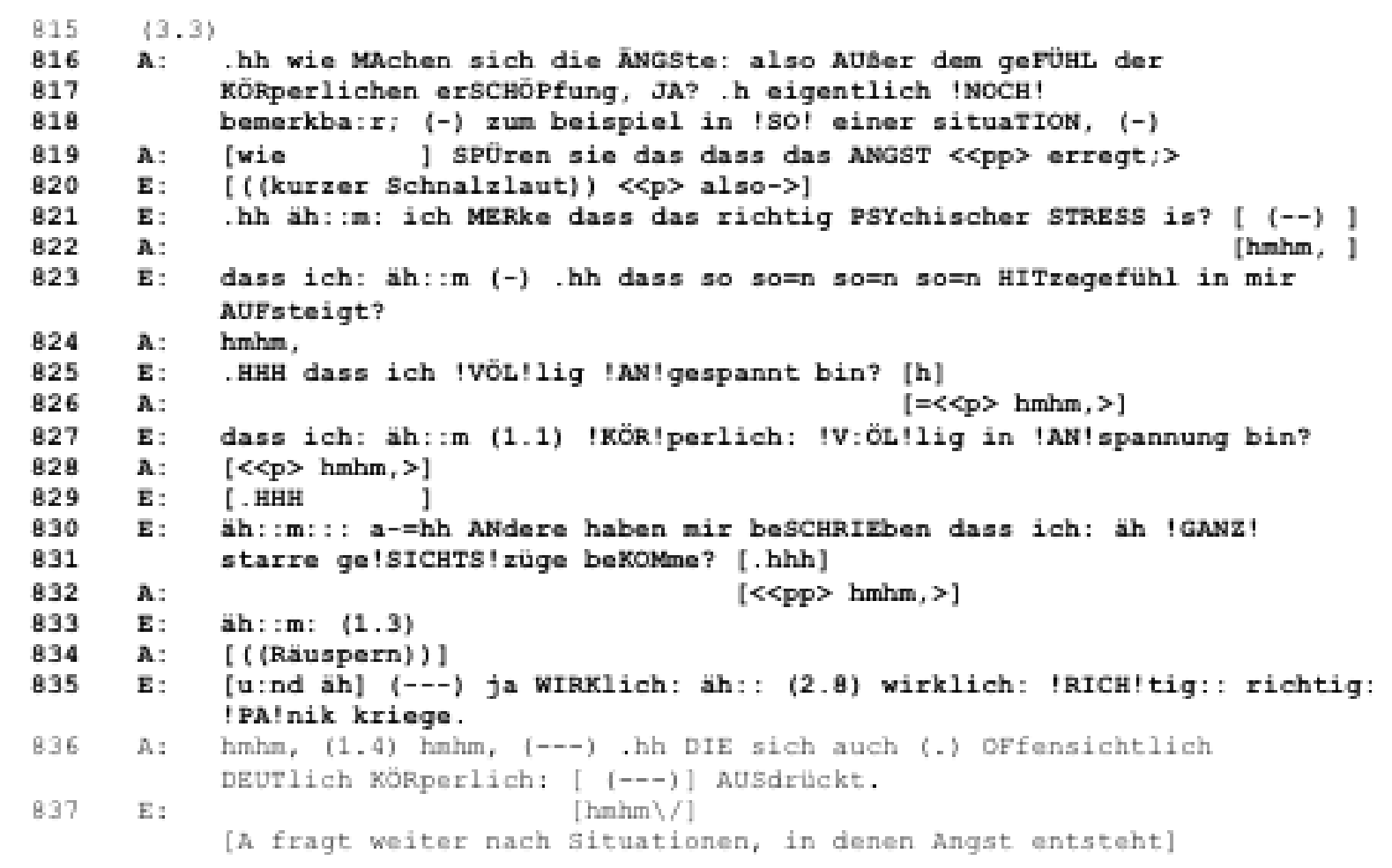

Ausschnitt 3: wirklich: !RICH!tig:: richtig: !PA!nik kriege - Frau Epang, Z. 815-839

\section{Sprechersiglen: $\mathbf{E}=$ Frau Epang; $\mathbf{A}=$ Arzt 2}

Frau Epang beginnt bereits in Überlappung mit der Frage des Arztes ihren Turn mit also, wartet dann zunächst ab, bis der Arzt seine Frage beendet hat, und beginnt anschließend mit einem ersten Listenelement direkt eine Aufzählung dessen, wie sich Ängste "noch bemerkbar machen". Sie behandelt damit die Frage des Arztes als Projektionskomponente (Z. 816-821), und zwar in Form einer Prä-Detaillierungskomponente (s. o. Abschnitt 1.1), indem sie ihre Aufzählung relevanter Merkmale direkt an die Äußerung des Arztes anschließt. Die Gesamtkonstruktion wird hier also ko-konstruiert, was lexikalisch am ersten Listenelement erkennbar ist (bemerkba:r - ich MERke).

Die folgenden fünf Turns sind prosodisch recht gleichförmig als Listenelemente gestaltet: Sie beginnen jeweils mit Verzögerungssignalen (ähm, hörbares Einatmen) und enden mit stark steigender Tonhöhe. Die Akzentsetzung trägt zu dieser Gleichförmigkeit bei, wobei die letzten drei der fünf Listenelemente deutlich stärker akzentuiert sind. Der Arzt ratifiziert jedes Listenelementemit einem Hörersignal (Z. 821-832).

Mit dem ersten Listenelement nimmt Frau Epang lexikalisch Bezug auf die Frage des Arztes und nennt zunächst einen nicht besonders spezifischen psychischen Aspekt (Z. 821). Im zweiten Element beginnt sie dann mit der Aufzählung körperlicher Phänomene (Z. 823-824); das dritte Anzeichen für Angst wird im vierten Listenelement präzisierend reformuliert (!VÖL!lig !AN!gespannt, !KÖR!perlich: !V:ÖL!lig in !AN!spannung, Z. 825, 827). Das fünfte Listenelement ist um den Zusatz erweitert, dass Frau Epang das beschriebene körperliche Symptom als Beobachtung von nicht genannten anderen einführt; prosodisch ist es aber deutlich als Teil der Auflistung erkennbar (Z. 830-831). 
Nach einem Verzögerungssignal in Form einer gefüllten Pause schließt Frau Epang die Listenkonstruktion ab: Sie setzt mit einem additiven Anschluss an, zögert erneut (äh) und fährt nach einer Pause hochstufend mit ja wirklich fort, macht dann aber erneut eine längere Pause. Diese Darstellung von Formulierungsarbeit deutet auf Wortsuche und Schwierigkeiten beim Beschreiben bzw. Benennen des Zustandes hin. Nach diesen Verzögerungen verwendet Frau Epang die konventionell vorgeformte Wendung "(die) Panik kriegen"9. Durch die Doppelung des Attributs richtig, Dehnungen und starke Akzentuierung stuft sie die lexikalisch und phraseologisch bereits als extrem formulierte Angst weiter hoch. Dass dies kein weiteres Listenelement ist, wird zum einen an der allgemeinen Qualität des Ausdrucks erkennbar, zum anderen an der tief fallenden Intonation.

Schematisch kann diese Listenkonstruktion wie folgt dargestellt werden:

\begin{tabular}{|c|c|c|c|}
\hline \multicolumn{2}{|c|}{$\begin{array}{l}\text { I. Projektionskomponente } \\
\text { (Frage des Arzles) }\end{array}$} & $\begin{array}{l}\text { A: hh wie MAchen sich die ANGSie: also AUßer dem } \\
\text { geFOHL der KÖRperlichen erSCHOPfiung. JA? .h }\end{array}$ & \\
\hline \multirow[t]{5}{*}{ II. Liste } & 1. Listenelement & $\begin{array}{l}\text { [ }<\langle p\rangle \text { also- }>\text { J hh ah :-m: ich MERke dass das richtig } \\
\text { PSYchischer STRESS is? }\end{array}$ & \\
\hline & 2. Listenelement & $\begin{array}{l}\text { dass ich: äh::m }(-) \text {. hh dass so } s o=n \text { so }=n \text { so }=n \\
\text { HIT zegefibh in mir AUFsteigl? }\end{array}$ & \\
\hline & 3. Listenelement & HHH dass ich!VOL!lig!AN!gespannt bin? & \\
\hline & 4. Listenelement & $\begin{array}{l}\text { dass ich: äh::m (l.I) !KOR!perlich: IV:OLIlig in } \\
\text { IAN!spannung bin? }\end{array}$ & \\
\hline & 5. Listenelement & 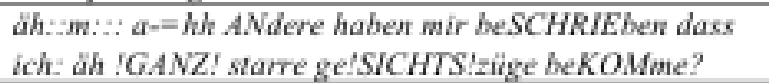 & \\
\hline \multicolumn{2}{|c|}{ III. GestaltschlieBungskomponente } & $\begin{array}{l}\text { [u:nd àh] (--) ja WIRKlich: äh:: (2.8) wirklich: } \\
\text { IRICH!tig:! richtig: !PA!nik kriege. }\end{array}$ & $\begin{array}{l}\text { vorgeformter } \\
\text { Ausdruck (_Panik } \\
\text { kriegen“) }\end{array}$ \\
\hline
\end{tabular}

Schematische Darstellung 3: Listenkonstruktion wirklich: !RICH!tig:: richtig: !PA!nik kriege (Frau Epang)

Die Ko-Konstruktion der Gesamtstruktur wird zum einen durch den direkten Anschluss des ersten Listenelements an die Frage des Arztes und zum anderen durch die lexikalische Wiederaufnahme erkennbar.

Zudem ist an diesem Beispiel zu beobachten, wie sich die Gesprächsbeteiligten an einem vorgeformten Ausdruck orientieren, um eine Listenkonstruktion, und damit die gesamte Sequenz und das Thema abzuschließen: Der Arzt ratifiziert mit zwei vokalen Hörersignalen und nimmt die Verallgemeinerung zurück, indem er den Turn von Frau Epang mit einem Relativsatz erweitert und in einer Zusammenfassung der von Frau Epang in der Liste genannten Punkten die !PA!nik spezifiziert als eine, DIE sich auch (.) OFfensichtlich DEUTlich KÖRperlich: [ (---)] AUSdrückt (Z. 833-836). Frau Epang ratifiziert dies mit einem Hörersignal (Z. 837), woraufhin der Arzt das Thema verschiebt und Rückfragen zu Situationen stellt, in denen eine solche Angst auftritt. Hier wird also auch deutlich, wie ein

\footnotetext{
${ }^{9}$ Die Wendung "die Panik kriegen" ist in phraseologischen Wörterbüchern und Datenbanken wie redensarten-index.de nicht verzeichnet, sie findet sich aber als umgangssprachliche Wortkombination in allgemeinen Lexika (z. B. Duden Online). Bei "Panik kriegen" handelt es sich entweder um eine Anspielung darauf oder um eine - schwach vorgeformte umgangssprachliche Kollokation bzw. einen "geprägten Ausdruck" (Feilke 1996: 91-95), ein "idiom of encoding" (Makkai 1972: 24, 57).
} 
vorgeformter Ausdruck, der einen bündelnden, generischen oder generalisierenden Effekt hat, die Funktion übernimmt, die Listenkonstruktion $\mathrm{zu}$ beenden; er eignet sich also für die funktional relevante Position der Gestaltschließungskomponente. Vorgeformte Ausdrücke können auch an den anderen funktional relevanten Stellen von Listenkonstruktionen stehen, nämlich als Projektionskomponente und als letztes Listenelement (cf. Knerich i. Vorb.).

\section{$4 \quad$ Listenkonstruktionen mit inszenierender Funktion}

Bestimmte vorgeformte Ausdrücke als Listenelemente können dazu dienen, inhaltlich nichtdetaillierende Listen zu konstruieren, die vor allem die Funktion der Inszenierung haben. Im hier zugrunde gelegten Korpus verwenden PatientInnen Listenkonstruktionen, die als strukturell vorgeformte Konstruktion formal nahelegen, dass detailliert wird, während ihre Elemente zumindest zum Teil inhaltlich bzw. semantisch leer oder stark reduziert sind. Für die Formulierung solcher leeren oder vagen Äußerungsteile nutzen die InteraktantInnen vorgeformte Wendungen oder Ausdrücke, die konventionell für eine Vielzahl von Elementen oder Aspekten stehen, ohne diese näher zu benennen.

Eine solche Listenkonstruktion gebraucht Frau Wiesinger in der folgenden Sequenz. Der Ausschnitt stammt aus der offenen Anfangsphase des Gesprächs und bezieht sich auf die Unterscheidung zwischen organischen und psychischen Erkrankungen, die angesichts der körperlichen Symptome der Angsterkrankung für Frau Wiesinger nicht einfach ist. Der erste hier dargestellte Turn nimmt Bezug darauf, dass noch nicht durch einen Allergietest abgeklärt werden konnte, ob das Gefühl zu ersticken eventuell doch organische Ursachen hatte.

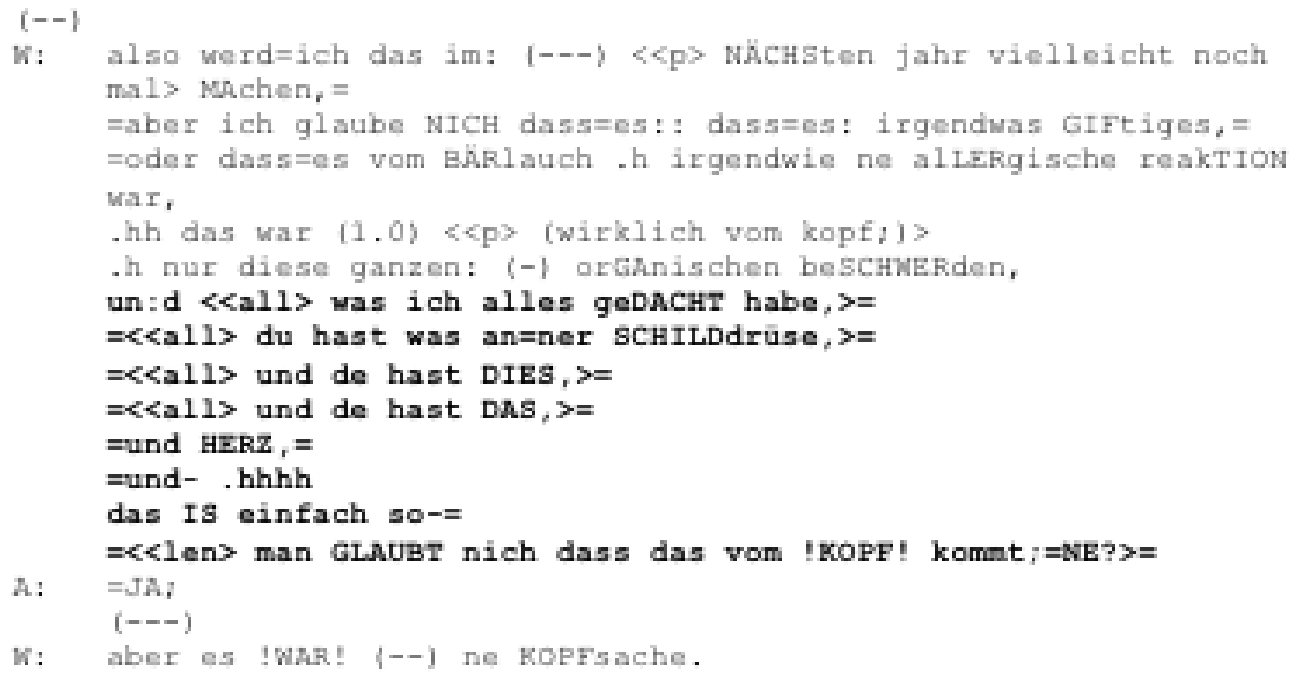

Ausschnitt 4: und de hast DIES, und de hast DAS - Frau Wiesinger, Z. 327-345

\section{Sprechersiglen: $\mathbf{W}$ = Frau Wiesinger; $\mathbf{A}=$ Arzt 1}

Frau Wiesinger verweist auf die starken Paniksymptome, die an dieser Stelle bereits eingeführt sind, hier nur noch mit es; der bestimmte Artikel das in der vorgeformten Wendung das war (1.0) $<<p>$ wirklich vom kopf; $>$ (Z. 333) bezieht sich anaphorisch auf die in der vorhergehenden Erzählung als dramatisch dargestellte Angst im Rahmen der Panikerkrankung (zu "das war vom Kopf" und "das war ne Kopfsache" als vorgeformte 
Wendungen bei der Formulierung von Aspekten einer subjektiven Krankheitstheorie cf. Knerich i. Vorb.).

In Z. 334 referiert Frau Wiesinger elliptisch auf die orGAnischen beSCHWERden, die sie in der Zeit hatte, und schließt additiv eine Referenz auf damit zusammenhängende Annahmen an, die als Projektionskomponente in Form einer Prä-Detaillierungskomponente fungiert: Mit dem kataphorischen Element alles und der steigenden Intonation werden die folgenden Listenelemente projiziert.

Mit den anschließenden fünf Elementen listet Frau Wiesinger Erkrankungen auf, die sie früher als Erklärungen für die "organischen Beschwerden" in Betracht gezogen hat. Die ersten drei Listenelemente sind als Gedankenwiedergabe bzw. Wiedergabe eines inneren Dialogs in der zweiten Person Singular formuliert, das vierte besteht aus einem Wort, das fünfte ist leer. Mit dem ersten Listenelement formuliert sie einen möglichen Erkrankungsherd (was an=ner SCHILDdrüse, Z. 336). Dann folgt eine vorgeformte Wendung über zwei Listenelemente (und de hast DIES, ==und de hast DAS, Z. 337-338) ${ }^{10}$. Diese beiden Elemente sind also inhaltlich leer. Die Demonstrativpronomen verweisen nicht auf etwas in der umgebenden Rede; vielmehr wird die große Menge beziehungsweise die Vielfalt der in Betracht gezogenen Erkrankungen dargestellt, ohne diese zu benennen. Das vierte Listenelement ist elliptisch, es besteht aus der additiven Konjunktion und und dem Wort HERZ (Z. 339). Das fünfte, abschließende Listenelement besteht nur noch aus der additiven Konjunktion in schwebender Intonation bleibt dann leer bzw. offen ${ }^{11}$, anstatt der Nennung eines weiteren möglicherweise erkrankten Organs oder der Formulierung eines generalisierenden Abschlusselements (cf. Jefferson 1990, s. o.) folgt ein deutlich hörbares, langes Einatmen. Auch dieses leere letzte Element stellt die Vielzahl der unzutreffenden möglichen Erklärungen und Diagnosen dar.

Für den ersten Teil der Gestaltschließungskomponente und als Gelenkstelle zum nächsten Turn verwendet Frau Wiesinger eine vorgeformte Struktur (das IS einfach so-, Z. 341) und fährt dann in schnellem Anschluss mit der Negation des Glaubens an die vorher genannte und für sie heute gültige Erklärung fort: $=<<l e n>$ man GLAUBT nich dass das vom !KOPF! kommt $;=N E ?>=(\mathrm{Z} .342)$. Diese Erklärung markiert sie auch stimmlich durch die langsame Sprechweise und die starke Akzentsetzung, womit sie ihre damaligen Zweifel an dieser Erklärung betont. Hier stimmt der Arzt sehr emphatisch zu, übernimmt aber nicht das Rederecht, woraufhin Frau Wiesinger nach einer Gap die jetzt gültige Erklärung formuliert: aber es !WAR! (-) ne KOPFsache.. Der zweite Teil der Gestaltschließungskomponente rahmt somit nicht nur die Listenkonstruktion, sondern weist anaphorisch auf den Beginn dieser Sequenz zurück (Z. 333).

Schematisch kann diese Listenkonstruktion wie folgt dargestellt werden:

\footnotetext{
10 "Dies und das" ist als vorgeformte Wendung in entsprechenden Wörterbüchern verzeichnet und wird z. B. mit "mancherlei" umschrieben, d.h. der Ausdruck selbst steht für eine Vielzahl von Elementen oder Aspekten, ohne diese näher zu benennen (cf. Duden - Redewendungen 2002: 166).

${ }^{11}$ Für eine genaue Beschreibung der Aposiopese als einer solchen leeren bzw. offenen syntaktischen Konstruktion vgl. Imos Arbeit zu Aposiopese-Konstruktionen (Imo 2011).
} 


\begin{tabular}{|c|c|c|c|}
\hline \multicolumn{2}{|c|}{ I. Projektionskomponente } & un: $d<<$ all $\rangle$ was ich alles geDACHT habe, $>$ & \\
\hline \multirow{5}{*}{$\begin{array}{l}\text { II. } \\
\text { Liste }\end{array}$} & 1. Listenelement & $=<<a l l>$ du hast was an=ner SCHILDdrüse, $>=$ & \multirow{5}{*}{$\begin{array}{l}\text { vorgeformter Ausdruck } \\
\text { (_dies und das") }\end{array}$} \\
\hline & 2. Listenelement & $=<<a l l>$ und de hast DIES, $>=$ & \\
\hline & 3. Listenelement & $=\langle<a l l>$ tund de hast $D A S\rangle=$ & \\
\hline & 4. Listenelement & $=$ und $H E R Z,=$ & \\
\hline & 5. Listenelement & $=$ und - , hhbh & \\
\hline \multicolumn{2}{|c|}{ III. Gestaltschließungskomponente } & $\begin{array}{l}\text { das IS einfach so- }==<<\text { len }>\operatorname{man} \text { GLAUBT } \\
\text { nich dass das vom !KOPF! kommt; }=N E ?>=\end{array}$ & $\begin{array}{l}\text { vorgeformter Ausdruck (,das } \\
\text { kommt vom Kopf }{ }^{\text {s) }}\end{array}$ \\
\hline
\end{tabular}

Schematische Darstellung 4: Listenkonstruktion und de hast DIES, und de hast DAS (Frau Wiesinger)

Auffällig an dieser Liste ist, dass hier die große Anzahl möglicher Elemente und die Offenheit der Liste mit verschiedenen Mitteln dargestellt wird: Es werden mehr als die für Listenkonstruktionen präferierten drei Elemente genannt; eine vorgeformte Wendung, die zwei Demonstrativpronomen enthält, verweist auf eine Unzahl weiterer Gedanken an mögliche Erkrankungen; das letzte Element bleibt lexikalisch leer und besteht nur aus einem tiefen Atemzug. Der vorgeformte Ausdruck und de hast DIES, und de hast DAS, wird auseinandergezogen und füllt so zwei Listenelemente, zudem steht er in einer "Stellvertreterfunktion" für etwas, das weder hier noch später genannt wird.

Es gibt im Korpus noch weitere Beispiele für die Darstellung von Detaillierung ohne Nennung inhaltlicher Details, die dem hier betrachteten vergleichbar sind. Unter anderem sind sie ebenfalls mit Absolutheitsformulierungen eingeleitet, und die Sprecherinnen verwenden die vorgeformten Ausdrücke "das und das" oder "dies und das". Dies ist ein Hinweis darauf, dass diese Art der Listenkonstruktion in diesem Kontext musterhaft verwendet wird.

Die innerhalb dieser Konstruktionen verwendeten sozial geteilten vorgeformten Ausdrücke "das und das" und "dies und das" weisen in ihrer konventionellen idiomatischen Bedeutung auf eine große Menge beziehungsweise eine nicht genannte oder nicht nennbare Vielfalt von Elementen hin. Ihr Gebrauch in dieser Stellvertreterfunktion unterscheidet sie von dem Gebrauch in einer "Platzhalterfunktion", wie Lehnen (2000) ihn für kollaborative Textproduktion rekonstruiert. Danach verwenden InteraktantInnen derartige vorgeformte Wendungen bei der gemeinsamen Textproduktion als Ersatz für Inhaltselemente (cf. Lehnen 2000: 51, 64; 227-230). Der Unterschied zu der hier gezeigten Verwendung ist, dass Platzhalter beim gemeinsamen Schreiben entweder für bereits produzierte, aber für den aktuellen Arbeitsschritt nicht relevante Inhalte stehen oder für noch zu produzierende Inhalte, die später nachgeliefert werden. Im Fall der nichtdetaillierenden Listen dagegen werden für die vorgeformten Ausdrücke in Stellvertreterfunktion keine Inhaltselemente ergänzt, sie bleiben Leerstellen.

\section{$5 \quad$ Fazit}

In diesem Beitrag wurden Listenkonstruktionen anhand von Ausschnitten aus authentischer Kommunikation als vorgeformte Strukturen rekonstruiert, die von den umgebenden Äußerungen klar abgrenzbar sind und sich durch eine verfestigte Oberflächenstruktur auszeichnen, die vor allem über die Prosodie angezeigt wird. Sie bestehen aus einer Aufzählung von $n$ Elementen, die von einem einleitenden und einem abschließenden Element gerahmt ist. Zwischen den rahmenden Elementen besteht ein inhaltlicher Bezug, zudem werden die Listenelemente semantisch und zumeist syntaktisch parallel gestaltet. Die Funktion solcher Listenkonstruktionen ist in den meisten Kontexten das Detaillieren. Im hier 
untersuchten Korpus finden sich aber auch Listenkonstruktionen mit inszenierender Funktion, in denen wenig oder gar nicht detailliert wird bzw. das Detaillieren nur angedeutet wird. Um $\mathrm{zu}$ inszenieren, verwenden die SprecherInnen bei der Produktion solcher Listenkonstruktionen weitere, vor allem prosodische und lexikalische Mittel. Innerhalb dieser Konstruktion dienen vorgeformte Ausdrücke, die konventionell eine Vielzahl von Aspekten ausdrücken, als zusätzliche Ressource.

Bei der Betrachtung solcher Listenkonstruktionen in authentischen Gesprächen fällt auf, dass Interagierende sich an dieser Struktur orientieren, sie aber nicht immer in derselben schematischen Weise produzieren; z. B. passen sie sie ihren kommunikativen Zwecken an, indem sie die Anzahl der Listenelemente erweitern. Dies wurde auch für Phraseologismen im engeren Sinne beobachtet: Im Interaktionsprozess geht es offenbar nicht darum, die korrekte lexikalisierte - Form zu finden, sondern es finden sich Abweichungen, individualisierte Formen, Anspielungen etc. (cf. Dausendschön-Gay et al. 2007: 205-6, 209, Knerich i. Vorb., Quasthoff 1983: 8, Schmale 2011: 182).

Die jeweilige Einbettung der untersuchten Listenkonstruktionen in den weiteren Gesprächskontext konnte hier nur am Rande betrachtet werden, sie ist aber für eine Auswertung der Gespräche im medizinischen Kontext (cf. Gülich/Schöndienst 1999, Gülich 2007) interessant: Listenkonstruktionen können innerhalb größerer Einheiten verwendet werden, z. B. in narrativen Rekonstruktionen (Ausschnitt 4), in allgemeinen Darstellungen (Ausschnitt 2) oder in Bewertungssequenzen (Ausschnitt 1), sie können aber auch als Antwort auf eine Frage für sich stehen (Ausschnitt 3). Bei der Bearbeitung der kommunikativen Aufgabe der Detaillierung und auch der Inszenierung können InteraktantInnen unter anderem zwischen narrativen Darstellungsformen und Listenkonstruktionen wählen. Die Auswertung des vorliegenden Korpus hat ergeben, dass PanikpatientInnen in einer bestimmten sequenziellen Umgebung, nämlich wenn der Arzt/die Ärztin Zugzwänge setzt, die auf das Erzählen bestimmter Episoden oder bestimmter situativer Elemente abzielen, Listenkonstruktionen gegenüber einer narrativen Rekonstruktion bevorzugen und damit detailliertere Ausführungen umgehen. Bei PatientInnen mit epileptischen Anfallserkrankungen ist dies im untersuchten Korpus nicht der Fall.

Hinsichtlich der Einordnung von größeren verfestigten Einheiten hat sich noch kein eindeutiges Konzept herausgebildet; es gibt aber derzeit mehrere Konzepte und Begriffe, die fest geprägte Phänomene unterschiedlicher Größe und auch Muster, die auf unterschiedlichen Ebenen verfestigt sind, erfassen. Günthner (2006: o. S.) verwendet "Vorgeformtheit" als Oberbegriff, unter den verfestigte Einheiten von grammatischen Konstruktionen über Phraseologismen bis zu kommunikativen Gattungen fallen. Schmale bezieht in seinem Vorschlag zur Definition der "präformierten Konstruktionseinheit" ebenfalls kommunikative Gattungen ein (cf. Schmale 2011: 180): Solche Einheiten bestehen ihm zufolge aus "mehr oder weniger fest kombinierten lexikalischen, prosodischen, nonverbalen, situativkontextuellen, sozialen, textuellen usw. Faktoren"; sie können "unterschiedliche Transparenzgrade" besitzen und sowohl rein individuell als auch in Gruppen verschiedener Größe verwendet werden (cf. Schmale 2011: 188). 
Im Rahmen des Konzepts der vorgeformten Strukturen wird dagegen diskutiert, ob kommunikative Gattungen aus theoretischen und heuristischen Gründen überhaupt in das Konzept einbezogen werden sollten, da sie nicht nur auf der Ebene der sprachlichen Oberfläche verfestigt sind. Dausendschön-Gay et al. (2010) zählen kommunikative Gattungen zu den als vorgeformte Strukturen zur Verfügung stehenden Formulierungsressourcen. Quasthoff (2010) dagegen weist darauf hin, dass die Konzepte "Vorgeformtheit" und "kommunikative Gattung" voneinander unterschieden werden müssen, obwohl im Grundsatz für beide Konzepte gilt, dass rekurrente Aufgaben $\mathrm{zu}$ rekurrenten Lösungen führen. Sie bezieht Vorgeformtheit rein auf die Ebene der sprachlichen Form, während sie das Konzept der kommunikativen Gattungen von Bergmann und Luckmann (1995) als bezogen "auf Strukturprinzipien, Regelmäßigkeiten im Aufbau sprachlicher Sequenzen, Handlungsmuster, also funktionell bestimmte Strukturierungsvorgaben für die Lösung jeweils bestimmter Typen von 'gesellschaftlichen Problemen'" ansieht (Quasthoff 2010: 20). Diese können einen unterschiedlichen Grad an Festigkeit haben, dabei sind jedoch nicht kommunikative Gattungen an sich vorgeformt, sondern es gibt Gattungen, die vorgeformt oder gar formelhaft realisiert werden, und solche, die weniger stark vorgeformt realisiert werden bzw. für deren Realisierung auf der Formebene zwischen verfestigten und freien Formulierungen gewählt werden kann (vgl. Quasthoff 2010):

"Auch sehr rigide Baumuster und feste soziolinguistische Normen begründen [...] noch keine Formelhaftigkeit der gesamten Einheit, sondern lediglich die Gattungsorientierung, Formelhaftigkeit ist eine zusätzliche Eigenschaft der sprachlichen Formulierungsverfahren bzw.

Ressourcen, die allerdings fest an Gattungen gebunden sein können." (Quasthoff 2010: 20)

Auch in dieser engeren Fassung des Konzepts der Vorgeformtheit würden Listenkonstruktionen noch $\mathrm{zu}$ den vorgeformten Strukturen zählen, sie wären aber ein Randphänomen. Insgesamt ist festzustellen, dass es zwischen stark verfestigten - geradezu formelhaften - Einheiten und weniger stark verfestigten kommunikativen Aktivitäten viele Gemeinsamkeiten gibt. Wenn man authentische Gespräche betrachtet, ist zudem eher eine Kontinuität zwischen prototypischen Phänomenen und Randphänomenen zu beobachten (cf. auch Gülich/Kraft 1998). Dennoch legt die Breite der verfestigten Phänomene die Notwendigkeit einer internen Differenzierung nahe. Ich habe hier zwischen vorgeformten Ausdrücken, Wendungen und vorgeformten Strukturen unterschieden. Damit habe ich verfestigte Wortkombinationen auf Satz-, Turn- oder Ausdrucksebene abgegrenzt von auf der sprachlichen Oberfläche verfestigten Einheiten, die mehr als eine TCU oder einen Satz umfassen. Vorgeformtheit könnte dann als übergreifendes Konzept hinzukommen, das neben vorgeformten Wendungen, Ausdrücken und Strukturen auch kommunikative Gattungen oder Textmuster beinhaltet.

\section{Literatur}

Bergmann, Jörg R. (1994): "Ethnomethodologische Konversationsanalyse". In: Fritz, Gerd/Hundsnurscher, Franz (eds.): Handbuch der Dialoganalyse. Tübingen, Niemeyer: 316.

Bergmann, Jörg R./Luckmann, Thomas (1995): "Reconstructive Genres of Everyday Communication". In: Quasthoff, Uta M. (ed.): Aspects of Oral Communication. Berlin, de Gruyter: 289-304. 
Burger, Harald (1982): "Klassifikation: Kriterien, Probleme, Terminologie". In: Burger, Harald/Buhofer, Annelies/Sialm, Ambros (eds.). Handbuch der Phraseologie. Berlin/New York, de Gruyter: 20-56.

Burger, Harald (1998): Phraseologie. Eine Einführung am Beispiel des Deutschen. Berlin: Erich Schmidt Verlag.

Burger, Harald et al. (2007): "Phraseologie: Objektbereich, Terminologie und Forschungsschwerpunkte". In: Burger, Harald et al. (eds.): Phraseologie / Phraseology. Ein internationales Handbuch zeitgenössischer Forschung/An International Handbook of Contemporary Research. (= Handbücher zur Sprach- und Kommunikationswissenschaft/Handbooks of Linguistics and Communication Science: 28/1). Berlin/New York, de Gruyter: 1-10.

Dausendschön-Gay, Ulrich/Gülich, Elisabeth/Uhmann, Susanne (2010): "Formulieren". Unveröffentlichtes Arbeitspapier der ZiF-Kooperationsgruppe Der Fall als Fokus professionellen Handelns.

Dausendschön-Gay, Ulrich/Gülich, Elisabeth/Krafft, Ulrich (2007): "Vorgeformtheit als Ressource im konversationellen Formulierungs- und Verständigungsprozess". In: Hausendorf, Heiko (ed.): Gespräch als Prozess. Linguistische Aspekte der Zeitlichkeit verbaler Interaktion. Tübingen, Narr: 181-219.

Duden (2002): Duden - Redewendungen. Wörterbuch der deutschen Idiomatik. 2., neu bearbeitete und aktualisierte Auflage. Mannheim: Dudenverlag.

Duden Online. URL: www.duden.de/suchen/dudenonline/die\%20Panik\%20kriegen, Stand: 30.11.2012.

Feilke, Helmuth (1996): Sprache als soziale Gestalt. Ausdruck, Prägung und die Ordnung der sprachlichen Typik. Frankfurt/M.: Suhrkamp.

Gülich, Elisabeth (2007): "'Volle Palette in Flammen'. Zur Orientierung an vorgeformten Strukturen beim Reden über Angst". In: Boothe, Brigitte (ed.): Erzähltes Alter - erzählte Angst. Themenheft Psychotherapie und Sozialwissenschaft, 9/1: 59-87.

Gülich, Elisabeth (1994): "Formulierungsarbeit im Gespräch". In: Čmejrková, Světla/Daneš, František/Havlová, Eva (eds.): Writing vs Speaking. Language, Text, Discourse, Communication. Proceedings of the Conference held at the Czech Language Institute of the Academy of Sciences of the Czech Republic, Prague, October 14-16, 1992. Tübingen, Narr: 77-95.

Gülich, Elisabeth/Krafft, Ulrich (1998): "Zur Rolle des Vorgeformten in Textproduktionsprozessen". In: Wirrer, Jan (ed.): Phraseologismen in Text und Kontext. Bielefeld, Aisthesis: 11-38.

Gülich, Elisabeth/Krafft, Ulrich (1997a): «Le rôle du 〈préfabriqué 〉 dans les processus de production discursive ». In: Martins-Baltar, Michel (ed.): La locution entre langue et usages. Fontenay-aux-Roses, ENS-Editions Fontenay/Saint-Cloud: 241-276.

Gülich, Elisabeth/Krafft, Ulrich (1997b): "Momo und die Phraseme. Zur Rolle des Vorgeformten in Emile Ajars La vie devant soi". In: Sabban, Annette (ed.): Phraseme im Text: Beiträge aus romanistischer Sicht: Studien zur Phraseologie und Parömiologie. Bochum, Brockmeyer: 97-132. 
Gülich, Elisabeth/Krafft, Ulrich (1992): "'Ich mag es besser' - Konversationelle Bearbeitung vorgeformter Ausdrücke in Gesprächen zwischen deutschen und französischen Sprechern". Fremdsprachen Lehren und Lernen 21: 65-87.

Gülich, Elisabeth/Schöndienst, Martin (1999): "'Das ist unheimlich schwer zu beschreiben.' Formulierungsmuster in Krankheitsbeschreibungen anfallskranker Patienten: differentialdiagnostische und therapeutische Aspekte". Psychotherapie und Sozialwissenschaft. Zeitschrift für qualitative Forschung 1: 199-227.

Günthner, Susanne (2012): "'Geteilte Syntax': Kollaborativ erzeugte dass-Konstruktionen". gidi Arbeitspapierreihe 43, 08/2012: 1-21.

Günthner, Susanne (2006): "Von Konstruktionen zu kommunikativen Gattungen: Die Relevanz sedimentierter Muster für die Ausführung kommunikativer Aufgaben". gidi Arbeitspapierreihe 1/08: 173-190.

Imo, Wolfgang (2011): "Cognitions are not observable - but their consequences are: Mögliche Aposiopese-Konstruktionen in der gesprochenen Alltagssprache". Gesprächsforschung - Online-Zeitschrift zur verbalen Interaktion 12: 265-300.

Imo, Wolfgang (2007): "Der Zwang zur Kategorienbildung: Probleme der Anwendung der Construction Grammar bei der Analyse gesprochener Sprache". Gesprächsforschung Online-Zeitschrift zur verbalen Interaktion 8: 22-45.

Jefferson, Gail (1990): "List-construction as a task and a resource". In: Psathas, George (ed.): Interaction Competence. Washington, D.C., University Press of America: 63-92.

Kallmeyer, Werner/Keim, Inken (1986). "Formulierungsweise, Kontextualisierung und soziale Identität. Dargestellt am Beispiel des formelhaften Sprechens". Zeitschrift für Literaturwissenschaft und Linguistik 64: 98-126.

Knerich, Heike (i. Vorb.): Vorgeformte Strukturen als Formulierungsressource beim Sprechen über Angst und Anfälle. Dissertation, TU Dortmund.

Lehnen, Katrin (2000): Kooperative Textproduktion. Zur gemeinsamen Herstellung wissenschafticher Texte im Vergleich von ungeübten, fortgeschrittenen und sehr geübten SchreiberInnen. Dissertationsschrift, Universität Bielefeld.

Makkai, Adam (1972): Idiom Structure in English. The Hague: Mouton.

Pomerantz, Anita (1986): "Extreme Case formulations: A way of legitimizing claims". Human Studies 9: 219-229.

Quasthoff, Uta M. (2010): "'Ich kann jetzt nich Äpfel und Birnen miteinander vergleichen, ne' - Vorgeformtheit als gattungs- und rahmenorientiertes sprachliches Formprinzip". In: Hinrichs, Nicole/Limburg, Anika (eds.): Gedankenstriche - Reflexionen über Sprache als Ressource. Für Wolfgang Boettcher zum 65. Geburtstag. Tübingen, Stauffenburg: 13-32.

Quasthoff, Uta M. (1983): "Formelhafte Wendungen im Deutschen: Zu ihrer Funktion in dialogischer Kommunikation". Germanistische Linguistik 5-6/81: 1-24.

Sacks, Harvey/Schegloff, Emanuel A./Jefferson, Gail (1974). "A simplest systematics for the organisation of turn-taking for conversation". Language 50/4: 696-735.

Selting, Margret (2004): "Listen: Sequenzielle und prosodische Struktur einer kommunikativen Praktik - eine Untersuchung im Rahmen der Interaktionalen Linguistik". Zeitschrift für Sprachwissenschaft 23: 1-46.

Selting, Margret (2000): "Berlinische Intonationskonturen: 'Die Treppe aufwärts' - nebst Vergleichen mit entsprechenden Hamburger Konturen". In: InLiSt 16: 1-73. 
Selting, Margret et al. (1998): "Gesprächsanalytisches Transkriptionssystem (GAT)". Linguistische Berichte 173: 91-122.

Schmale, Günter (1999): "'Ich bin bei uns in der Stadt in aller Munde!' 'So wie Bill Clinton?' Interaktive Behandlung vorgeformter Sequenzen in ausgewählten Talkshows". In: Bravo, Nicole Fernandez/Behr, Irmtraud/Rozier, Claire (eds.): Phraseme und typisierte Rede: Eurogermanistik 14. Tübingen, Stauffenburg: 159-172.

Schmale, Günter (2011): "Was ist in der Sprache 'vorgeformt'? Überlegungen zu einer erweiterten Definition sprachlicher Präformiertheit". In: Schäfer, Patrick/Schowalter, Christine (eds.): In mediam linguam. Mediensprache - Redewendungen Sprachvermittlung. Festschrift für Heinz-Helmut Lüger zum 65. Geburtstag. Landau, VEP: 177-190.

Tannen, Deborah (1987): "Repetition in conversation: Toward a poetics of talk". Language 63: 574-605. 